\title{
Des chiffres pertinents grâce à la collaboration des médecins
}

\author{
Jürg Schlup \\ Dr méd., président de la FMH
}

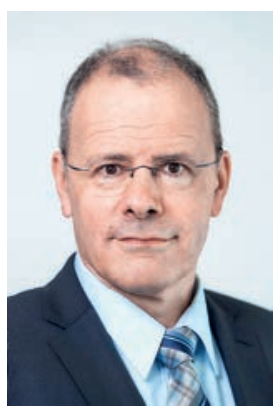

Bien que les revenus des médecins attirent régulièrement l'attention des médias et des politiques, les données disponibles sur ce sujet sont négligées [1]. Ainsi, le fait que plusieurs milliers de médecins aient fourni depuis 2016 des chiffres précis sur leur revenu à l'Office fédéral de la statistique (OFS) n'a pas empêché l'Office fédéral de la santé publique (OFSP) d'annoncer sa propre étude [2]. Promise pour fin 2017, la publication de l'OFSP se fait toujours attendre, alors que les résultats de l'OFS ont été publiés en avril 2018 [3].

Même si le relevé MAS de l'OFS n'a eu aucun écho médiatique ou politique - il n'a même pas été mentionné dans la réponse donnée à une interpellation parlementaire [4] - il mérite que l'on s'y attarde. En effet, grâce aux nombreuses données fournies par près de 7000 médecins, il apporte des informations détaillées sur les charges et les produits des cabinets médicaux et centres ambulatoires, ainsi que sur la proportion de leur revenu financée par les primes. En 2015, selon l'OFS, les cabinets constitués en entreprise individuelle ont dégagé un chiffre d'affaires moyen d'environ 545000 francs de leur activité professionnelle, dont 508000 francs de leur activité au cabinet [3]. Vu que les dépenses totales (frais de personnel et de matériel, charges locatives, etc.) représentent $72 \%$ du chiffre d'affaires, le résultat d'exploitation d'un cabinet individuel s'élève en moyenne à 155000 francs [3].

\section{Près de 7000 médecins ont fourni à l'OFS de} nombreuses données concernant leur chiffre d'affaires, leurs dépenses et leur résultat d'exploitation.

Si l'on considère que le chiffre d'affaires de l'activité au cabinet est généré à 89\% par l'assurance-maladie obligatoire, on peut conclure que la part du résultat issue des primes s'élève en moyenne à environ 138000 francs par an $(=155000 \times 0,89)$.

Afin de vérifier la fiabilité des résultats du relevé MAS, la FMH a comparé les données sur lesquelles celui-ci s'appuie à celles recueillies dans le cadre de l'étude

RoKo. Notre analyse (pages 1480 à 1481) montre que la sous www.bullmed.ch

$\rightarrow$ Numéro actuel ou

$\rightarrow$ Archives $\rightarrow 2018 \rightarrow 43$
Confédération et la FMH obtiennent des résultats comparables [5]. Cela confirme que les chiffres de l'OFS décrivent les revenus des médecins indépendants de manière réaliste.

Le relevé MAS représente une avancée majeure pour deux raisons par rapport aux chiffres publiés précédemment par la FMH et le bureau BASS. Premièrement, la population étudiée, à savoir exclusivement les cabinets médicaux et les centres ambulatoires, est définie clairement, ce qui permet d'éviter les biais des études antérieures, qui portaient sur tous les médecins facturant de manière indépendante. Les médecins-chefs et

Selon les chiffres de l'OFS, un cabinet individuel moyen réalise un résultat brut de 138000 francs à partir des primes.

les médecins dirigeants d'hôpitaux étaient donc inclus, alors qu'ils tirent une grande partie de leurs revenus de leur emploi à l'hôpital et non du tarif ambulatoire ou des primes. Par contre, les médecins-assistants et les chefs de clinique, qui représentent un tiers du corps médical, ne facturent pas de manière indépendante; toutes les discussions sur le revenu des médecins faisaient donc systématiquement l'impasse sur le tiers des médecins en exercice ayant le revenu le plus faible.

Deuxièmement, les données présentées ici montrent clairement comment les revenus sont générés et permettent aux assurés de savoir précisément combien d'argent le médecin installé en cabinet reçoit du financement solidaire par les primes. L'étude FMH-BASS se basant sur les revenus soumis à l'AVS, il n'était donc pas possible de calculer ce qui résultait des primes, de l'activité au cabinet ou des activités médicales en général. Ces données validées sur les revenus des médecins ambulatoires ne seront utiles que si l'on tire vraiment profit des informations disponibles, et non si l'on poursuit les polémiques sur des cas particuliers ou si l'on politise des chiffres douteux sur des revenus à sept chiffres [6]. La FMH s'engage volontiers dans une discussion équitable et basée sur des faits! 